[16] Gegenbaur. Manuel d'Anatomie comparée, 1874, Paris.

[17] Isidore Geoffrox SATNT-Hilatre. Sur un Bouc lactifère. Comptes rendus. de l'Acad. des Sciences, 1852, 34, 386.

[18] Guinard. Précis de Tératologie, 1893, 235.

[19] HALleR. Elementa physiologia, 7, 2-18.

[20] Нбмвоцdт. Voyage aux régions équinoxiales du Nouveau Continent. Relation historique, $1,376$.

[21] LesBre. Traité de Tératologie de l'Homme et des animaux domestiques, 1927, Paris.

[22] Makalik. Le lait de Bouc. Le Lait, 1922, 340.

[23] Menuts. Revue vétérinaire, 1912, 215.

[24] Henri Mrune Edwards. Leçons sur la physiologie et l'anatomie comparée. Paris, 1870. Appareil mammaire, 9, 125. Londres.

[25] Oven. On the Anatomy of Vertebrates. Mammuals, I868, 3, 760.

[26] Porcher. Lactation chez le Bouc. Le Lait, 1922, 289.

[27] Roвer. Letter concerning a man who give suck to a child. Philos trans, $1742,41,461,813$.

[28] Sohlossberger. Analyse der Milch eine Bock. Ann. der Chimie und Pharm., $1844,5,431$.

[29] Stravescu. Comptes rendus de la Soc. cent. de Méd. vét., 1925, 80.

[30] Vialleton. Eléments de Morphologie des Vertébrés, 1911, Paris.

[31] Wiederschiev. Manuel d'Anatomie comparée des Vertébrés. Traduction de la deuxième édition allemande, par Moquin TANdon, 1890.

\title{
RECHERCHES SUR LA CASÉINE
}

\author{
par Mlle J. BRIGANDO
}

Docteur ès Sciences de l'Université de Lyon,

Chef de Travaux à l'Institut des Recherches Agronomiques.

(Suite.)

\section{RECHERCHES PERSONNELLES.} présure.

Ces recherches ont porté sur la caséine-acide et la caséine-

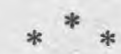

\section{CASÉINE-AGIDE.}

Notre but a été de déterminer les meilleures conditions dans lesquelles il faut se placer pour préparer une bonne caséine-acide, d'une teneur en cendres peu élevée, d'un bel aspect et d'une acidité faible.

Nous avons été amenée à examiner l'influence de la réaction du milieu, de l'agitation, de la température à laquelle la précipitation s'effectue, de la vitesse de l'addition de l'acide, de la concentration de ce dernier, du lavage. 
Ce simple exposé nous montre qu'il ne peut s'agir ici que de la préparation de la caséine par acidification provoquée. Nous nous sommes servie le plus souvent d'acide chlorhydrique comme agent précipitant, à la concentration de $2 \%$ environ.

Toutes les fois que nous faisons appel à un autre acide, nous le signalons.

Etudions maintenant, une à une, les eirconstances principales qui entourent la précipitation.

\section{RECHERCHES SUR LA RAPIDITÉ DE L'APPORT DE L'ACIDE DANS LA PRÉCIPITATION DE LA CASÉINE AU POINT ISOÉLECTRIQUE.}

Essai n०1. Précipitation rapide. - On opère ici sur des quantités de laboratoire : $250 \mathrm{~cm}^{3}$ de lait de $p$ H 6,5 et de $25^{\circ} \mathrm{D}$., en se servant d'acide ehlorhydrique N/2 comme agent précipitant. En moins de deux minutes, on ajoute $28 \mathrm{~cm}^{3}$ d'acide, soit $2 \mathrm{gr} .04$ par litre. Nous arrivons au $p \mathrm{H}=4,35$, dépassant done le point isoólectrique. La caséine est filtrée, pressée et séchée entre deux feuilles de papier-filtre.

Taux des cendres : $2,48 \%$.

Essai $n^{\circ} 2$ 2. - Un deuxième essai est effectué avec $250 \mathrm{~cm}^{3}$ de lait ; on ajoute, en moins de 1 minute, $27 \mathrm{~cm}^{3} \mathrm{~d}$ 'HCl $\mathrm{N} / 2$, soit $1 \mathrm{gr}$. 93 par litre de lait. Le $p \mathrm{H}$ du sérum est de 4,5. La caséine est pressée et séchée comme ei-dessus.

Taux des cendres : $2,49 \%$.

Essai no 3. Précipitation lente. (L'acide employé est toujours HCl N/2). - A $250 \mathrm{~cm}^{3}$ de lait de $20^{\circ}$ d'acidité D., on ajoute lentement l'acide. Après une demi-heure, $22 \mathrm{~cm}^{3} 8$ d'acide ayant été ajoutés, soit $1 \mathrm{gr}$. 66 par litre de lait, le $p$ H est de 4,8. On laisse une demi-heure au repos. La caséine est filtrée sur linge; le $p$ H du sérum $=5,17$. La caséine est pressée et séchée sur papierfiltre.

Taux des cendres : 2,50.

Essai no 4. - Dans un autre essai, toujours sur $250 \mathrm{~cm}^{3}$ de lait, l'addition d'acide demande deux heures. On ajoute $22 \mathrm{~cm}^{3} 8$ d'acide HCl et le $p$ H du sérum $=$ 5,05 . On continue l'addition d'acide pour arriver au $p \mathrm{H}=4,5$. Il a fallu $28 \mathrm{~cm}^{3} 8$ de HCl. La caséine est jetée sur linge et on la laisse s'égoutter toute la nuit. Le lendemain, elle est pressée et séchée entre du papier-filtre.

Cendres : $3,28 \%$.

Nous contrôlons toutes ces expériences par deux autres essais de précipitation lente et rapide, au point isoélectrique. Le lait a $19^{\circ}$ D. ; $p \mathrm{H}=6,85$. La concentration de $\mathrm{HCl}$ est de N/2.

Essai n ${ }^{\circ}$ 5. Précipitation lente. - A $250 \mathrm{~cm}^{3}$ de lait, on ajoute très lentement de l'acide, pour arriver ąu $p$ H $=5,10$, ee qui demande une demi-heure.

Dans toutes ces opérations, le liquide est agité mécaniquement. On continue l'agitation pendant deux heures, sans addition d'acide. Ensuite, on ajoute l'acide pour arriver au point isoélectrique. Il faut $24 \mathrm{~cm}^{3}$ d'acide, soit $1 \mathrm{gr}$. 75 par litre de lait, et on a un $p H=4,7$. La caséine est jetée sur linge, passée à la presse, séchée entre du papier-filtre.

Cendres : $2,33 \%$. 
Essai $n^{\circ}$ 6. Précipitation rapide du même lait. - On ajoute $25 \mathrm{~cm}^{3}$ d'acide en deux minutes, $p$ H du sérum $=4,7$. Le tout est laissé à la température du laboratoire, sans agitation. Le $p H$ du sérum remonte un peu : 4,78. On jette sur linge, on presse et on sèche entre du papier-filtre.

Taux des cendres : $2,27 \%$.

TABlead $\mathrm{X}$.

INFLUENGE DE LA RAPIDITÉ DE L'APPORT DE L'ACIDE SUR LE TAUX DE MINÉRALISATION DE LA CASÉINE.

\begin{tabular}{|c|c|c|c|c|}
\hline Essais & $\begin{array}{c}\text { Durée de } \\
\text { l'addition de } \\
\text { l'acide }\end{array}$ & $\begin{array}{c}\text { Acide } \\
\text { chlorhydri- } \\
\text { que en gr. } \\
\text { par litre } \\
\text { de lait }\end{array}$ & $\begin{array}{l}\text { pH du } \\
\text { sérum }\end{array}$ & $\begin{array}{l}\text { Cendres } \\
\text { pour } 100 \text { gr. } \\
\text { de caséine } \\
\text { anhydre }\end{array}$ \\
\hline $1 \ldots \ldots$ & moins de 2 minutes & 2,04 & 4,35 & 2,48 \\
\hline $2 \ldots \ldots \ldots$ & moins de 1 minute & 1,93 & 4,50 & 2,49 \\
\hline $3 \ldots \ldots \ldots \ldots$ & 1 heure & 1,68 & 5,17 & 2,50 \\
\hline $4 \ldots \ldots \ldots \ldots$ & 2 heures & 2,10 & 4,50 & 3,28 \\
\hline $5 \ldots \ldots \ldots \ldots$ & $1 / 2$ heure & 1,75 & 4,70 & 2,33 \\
\hline $6 \ldots \ldots \ldots \ldots \ldots \ldots$ & 2 minutes & 1,82 & 4,70 & 2,27 \\
\hline
\end{tabular}

Nous pensions, a priori, qu'il valait mieux que l'addition d'acide se fît lentement, pour la précipitation de la caséine au point isoélectrique. Les résultats obtenus ne confirment pas du tout notre supposition. La rapidité de l'apport de l'acide n'a pas d'influence sur le chiffre des cendres. Que nous l'ajoutions lentement ou rapidement, les taux de la minéralisation de la caséine sont voisins.

\section{INFLUENCE DE L'AGITATION.}

Essai n ${ }^{\circ}$ 7. Précipitation à la température ordinaire. Agitation vigoureuse. Apport lent d'acide. Gaséine non lavée. - La température est de $18^{\circ} \mathrm{C}$. Le lai a $19^{\circ}$ d'acidité ; son $p H=6,6$.

5 litres de lait écrémé sont agités dans une cuve émaillée à double fond. La vitesse de l'agitation est de 80 tours environ à la minute. L'apport de l'acide se fait très lentement.

-Après $3 / 4$ d'heure d'agitation ............

$\begin{array}{cc}\text { HCl ajouté } & \text { pH } \\ 405 & 4,85 \\ & 5,05 \\ 415 & 4,85 \\ 420 & 4,60\end{array}$

On a ajouté $420 \mathrm{~cm}^{3}$ d'acide chlorhydrique à 2 gr. $40 \%$, soit 2 gr. par litre de lait. - On note une régression du $p H$ vers l'alcalinité après le repos de 2 heures qui suit la première agitation. Pendant ce temps, l'acide a décalcifié lentement les sels sensibles à son action, notamment le caséinate de calcium. 
La caséine se trouve dans un état de division très accentué ; les flocons en sont fins ; on la jette sur toile et on la laisse égoutter toute la nuit. Le lendemain, sans la laver, on la passe à une presse à main et, graduellement, on arrive à une pression assez forte, ce qui demande 4 heures.

Lø gâteau recueilli est finement brisé, à la main, puis séché à l'étuve à $40^{\circ} \mathrm{C}$. On obtient un produit granuleux, mélange de grains fins et de grains plus grossiers dont la couleur est jaune ambrée.

Il est évident que si nous avions eu un moulin pour le gâteau de caséine pressée, nous aurions eu des grains plus réguliers. Ceci est d'ailleurs sans importance pour les observations à tirer de notre expérience.

Cendres calculées sur le produit anhydre : 2,90\%.

Rendement par litre de lait écrémé : $31 \mathrm{gr}$. 6 .

Essai no 8. Précipitation à la température ordinaire, Agitation très lente. Apport lent d'acide. Caséine non lavée. - On a opéré sur le même lait que dans l'essai précédent.

5 litres sont placés dans le bac. On verse l'acide très lentement, presque goutte à goutte ; l'agitation n'est pas vigoureuse ; elle se fait à la main et est juste suffisante pour disperser la goutte acide quand elle tombe dans la masse. Le point isoślectrique est facile à atteindre. La séparation de la caséine est nette et le sérum jaune ambré très clair : $p H=4,7$.

On abandonne pendant 2 heures. Le $p H$ remonte, comme dans l'essai précédent, mais un pou moins : $p H=4,8$.

La suite des opórations est la même que dans l'essai no 1 . Le volume de l'acide ajouté à $24 \%$ est de $395 \mathrm{~cm}^{3}$, soit, en poids, 1 gr. 89 d'acide chlorhydrique par litre de lait.

La caséine séchée à $40^{\circ}$ est un produit granulé, peu différent d'aspect du précédent, avec des grains un peu plus gros.

Cendres : $2,84 \%$.

Rendement : 31,6 gr. au litre.

TABLEAU XI.

INFLUENGE DE L'AGITATION SUR LE TAUX DE MINERALISATION DE LA CASÉINE,

\begin{tabular}{l|l|c|c|c|c}
\hline \hline Essais & Agitation & $\begin{array}{c}\text { Acide chlo- } \\
\text { rhydrique } \\
\text { en gr. par } \\
\text { litre de lait }\end{array}$ & $\begin{array}{c}\text { pH du } \\
\text { sérum }\end{array}$ & $\begin{array}{c}\text { Cendres } \\
\text { pour 100 gr. } \\
\text { de caséine } \\
\text { anhydre }\end{array}$ & $\begin{array}{c}\text { Rendement } \\
\text { par litre } \\
\text { de lait }\end{array}$ \\
\cline { 2 - 3 } & Vigoureuse & 2,00 & 4,60 & 2,90 & 31,60 \\
$8 \ldots \ldots \ldots$ & Lente & 1,89 & 4,80 & 2,84 & 31,60 \\
\hline \hline
\end{tabular}

Nous notons le même chiffre de cendres, le même rendement, en somme le même produit, que l'agitation soit vive ou modérée. 


\section{INFLUENCE DE LA TEMPÉRATURE.}

Essai n० 9. Précipitation à chaud. Caséine non lavée. — Le lait a $20^{\circ}$ d'acidité $\mathrm{D}$. Son $p \mathrm{H}=6,5$. On le porte à la température de $42^{\circ} \mathrm{C}$. La concentration de l'acide employé est de 2,4\%. L'agitation est réalisée mécaniquement à 80 tours à la minute. Nous avons suivi de très près la précipitation et nous avons noté que pour le $p H$ 5,3, la précipitation est à peu près complète. Le sérum est presque clair et la caséine se prend en masse.

On continue à ajouter l'acide pour arriver au $p H$ 4,6 et l'on obtient un produit compact très élastique.

Quantité d'acide ajouté pour les 5 litres : $200 \mathrm{~cm}^{3}$, soit $0 \mathrm{gr} .95$ d'acide par litre.

La caséine est jetée sur un linge, passée à la presse une demi-heure seulement, et le gâteau obtenu est.ensuite brisé à la main et séché à l'étuve à $40^{\circ} \mathrm{C}$. On obtient des grains d'une couleur jaune ambré, assez marquée.

Cendres : $4,36 \%$.

Rendement : 31,4 gr. au litre.

Si nous comparons cet essai-aux essais 7 et 8 précédents où la précipitation de la caséine a lieu à la température ordinaire, nous notons la grande influence de la température sur la quantité moindre d'acide nécessaire pour la précipitation, mais également - ce qui est plus important à nos yeux, - sur la forte minéralisation du produit résultant. Nous relevons d'autre part que la précipitation est quasi totale au $p$ H 5,3, auquel correspond, non pas une caséine entièrement déminéralisée, mais un caséinate calcique.

Essai no 10. Précipitation à chaud. Apport d'acide jusqu'à obtention d'un sérum clair. Caséine non lavée. - Dans cet essai, nous avons tenu à nous placer dans les mêmes conditions que l'industrie, sans nous inquiéter d'arriver exactement au point isoélectrique pour la précipitation de la caséine.

5 litres de lait sont chauffés dans une cuve à double fond, à la température de $42^{\circ}$. L'apport d'acide chlorhydrique à $2 \%$ se fait lentement. On agite à la main de temps en temps. Quand le sérum nous paraît elair, nous arrêtons l'opération. Le $p \mathrm{H}=5,20$.

On jette toute la masse sur une toile. La filtration est plus lente que dans les opérations antérieures et notamment plus que dans l'essai qui précède.

Il y a fortement lieu de penser, devant les allures si différentes de la précipitation, suivant que l'on opère à la température ordinaire ou à $40^{\circ}$, que l'élévation de la température favorise la précipitation en gros flocons, puis en masse, des caséinates les moins calciques. Ici, au $p H$ 5,2, tout le caillé est formé ; à la température ordinaire $\left(15^{\circ}\right.$ à $\left.20^{\circ}\right)$, au $p H$ 5,2, la floculation peut commencer, mais elle n'est pas très visible. Il suffit de chauffer sans modifier les autres conditions pour voir apparaitre des flocons, puis des masses. 
La caséine est, tout à Ia fois, élastique et spongieuse. On la passe à la presse à main. Elle est ensuite séchée à l'air sur du papier-filtre. Elle colle sur le papier et sur le linge.

Le volume d'acide employé est de $85 \mathrm{~cm}^{3}$ pour les 5 litres, soit $0 \mathrm{gr}$. 408 d'acide par litre de lait, chiffre très inférieur à celui de l'opération précédente, dans laquelle on étuit allé jusqu'au $p \mathrm{H} 4,6$.

Le produit sec est en grains compacts de couleur jaune ambrée.

Cendres : $4,20 \%$.

Rendement par litre : 31 gr.

Nous obtenons sensiblement le même chiffre de cendres que dans l'essai précédent, bien que nous ayons employé nettement moins d'acide. On en peut conclure que dans l'essai $n^{\circ} 9$, l'acide ajouté pour passer du $p \mathrm{H} 5,3$ - à ce moment, tout le caillé est formé et le sérum est clair - au $p \mathrm{H} 4,6, n^{\prime} a$ exercé nulle action décalcifiante sur le caillé. Il n'a agi que sur le système salin du sérum dont il a fait varier le $p \mathrm{H}$ vers une plus forte acidité.

\section{Essai no 11. Précipitation à $50^{\circ}$. Gaséine lavée.}

Le lait a $22^{\circ} \mathrm{D}$. On en prend 5 litres que l'on chauffe d̀ $50^{\circ}$. La concentration de l'acide employé est de $3 \%$. On ajoute l'acide lentement et, lorsque le sérum nous paraît clair, le $p$ H est de 5,47.

La caséine se trouve rassemblée en masse. La précipitation n'a demandé qu'un quart d'heure. On continue l'addition de l'acide pour atteindre le point isob́lectrique, et il arrive que nous le dépassons même, puisque nous arrivons au $p H=4,3$. On laisse au repos pendant 3 heures. Le $p$ H remonte très peu : 4,35 .

La caséine se trouve rassemblée en une masse compacte, au-dessus de laquelle est une partie plus pulvérulente qui a dû se formor avec le temps, la masse inférieure ayant été obtenue au début de l'opération. On décante le sérum et on lave deux fois avec l'eau de la conduite, qui est de l'eau calcaire. La filtration sur toile est diffícile. La caséine pulvérulente en bouche très rapidement les orifices. On laisse toute la nuit sur la toile et, le lendemain, la caséine est passée à la presse où elle est laissée 5 heures.

Quantité d'acide employée : $245 \mathrm{~cm}^{3}$.

Acide par litre : 1 gr. 47.

L9 prołuit séchá à $40^{\circ}$ est en grains compacts, pou teintés.

Taux des cendres : $5,40 \%$.

Rendement : 24,45 au litre.

Acidité : $0,76 \%$.

Nous notons une fois de plus et d'une manière extrêmement nette, la fâcheuse influence d'une température élevée dans la préparation de la caséine-acide. 
Tablead XII.

INFLUENCE DE LA TEMPERATURE DE PREGCIPITATION SUR LE TAUX DE MINÉRALISATION DE LA CASÉINE.

\begin{tabular}{|c|c|c|c|c|c|}
\hline Essais & $\begin{array}{l}\text { Tempé- } \\
\text { rature } \\
\text { de } \\
\text { précipi- } \\
\text { tation }\end{array}$ & $\begin{array}{c}\text { Acide } \\
\text { chlorhydri- } \\
\text { que en gr. } \\
\text { par litre de } \\
\text { lait }\end{array}$ & $\begin{array}{c}\text { pH } \\
\text { du } \\
\text { sérum }\end{array}$ & $\begin{array}{c}\text { Cendres } \\
\text { pour } 100 \text { gr. } \\
\text { de caséine } \\
\text { anhydre }\end{array}$ & $\begin{array}{l}\text { Rendement } \\
\text { par litre de } \\
\text { lait écrémé }\end{array}$ \\
\hline $7 \ldots \ldots \ldots \ldots$ & $18^{\circ}$ & 2,00 & 4,60 & 2,90 & 31,60 \\
\hline $8 \ldots \ldots \ldots$ & $18^{\circ}$ & 1,89 & 4,80 & 2,84 & 31,60 \\
\hline $9 \ldots \ldots, \ldots$ & $42^{\circ}$ & 0,96 & 4,60 & 4,36 & 31,40 \\
\hline $10 \ldots \ldots \ldots \ldots$ & $42^{\circ}$ & 0,408 & 5,20 & 4,20 & 31,00 \\
\hline $11 \ldots \ldots \ldots$ & $50^{\circ}$ & 1,47 & 4,35 & 5,40 & 24,45 \\
\hline
\end{tabular}

A $40^{\circ}$, nous avons eu par deux fois une précipitation presque totale de la caséine, au $p H=5,3$ sous une forme très minéralisée répordant, sans aucun doute, à un caséinate calcique.

Si l'on opère à $50^{\circ}$, la précipitation est complète à un $p \mathrm{H}$ plus élevé : 5,47 , c'est-à-dire à un point où le caséinate qui précipite doit être plus calcifié que le précédent; c'est ce que nous montre le dosage des cendres :

Précipitation à $40^{\circ}$. Taux de la minéralisation $\%: 4,30,4,20$.

$$
\text { à } 50^{\circ} \text {. — - — } \quad: 5,40 \text {. }
$$

Relevons, de plus, que la seconde caséine a été lavée.

Nous comprenons maintenant que le lavage ait peu d'efficacité ; il enlève partie des sels solubles de la trame du caillé, mais il ne peut enlever de la chaux au caséinate qui a précipité.

Précipitation par l'acide chlorhydrique d'un lait à différentes températures. - Un lait est précipité par l'acide chlorhydrique respectivement aux températures de: $0^{\circ}, 10^{\circ}, 20^{\circ}, 30^{\circ}$ et $40^{\circ}$. On a opéré sur de petites quantités de lait: $100 \mathrm{~cm}^{3}$, tandis que les expériences précédentes étaient effectuées sur plusieurs litres. Un premier essai nous indique qu'il faut $42 \mathrm{~cm}^{3}$ d'acide chlorhydrique $\mathrm{N} / 10$ pour $100 \mathrm{~cm}^{3}$ de lait à $0^{\circ}$, soit $1 \mathrm{gr}$. 53 par litre pour atteindre le $p H$ 4,8. Dans tous les essais, on ajoute la même quantité d'acide et l'on contrôle le $p H$ des liqueurs. La précipitation effectuée, tous les laits sont portés à $40^{\circ}$, pour être dans les mêmes conditions. La température de précipitation seule étant différente.

Essai n 012. Précipitation à $0^{\circ}$. - Lorsqu'on atteint le $p H=4,8$, que l'on contrôle d'abord approximativement avec le rouge de méthyle, puis avec le potentiomètre, on n'observe aucune précipitation; la liqueur est tout à fait homogène. 
Essai no 13. Précipitation à $10 \%$ - Après addition de l'acide, le lait de $p \mathrm{H} 4,8$ est également homogène.

Essai no 14. Précipitation à $20^{\circ}$. - On n'observe qu'une très fine précipitation au $p H$ 4,8, sans séparation nette de sérum.

Essai no 15. Précipitation à $30^{\circ}$. - On a, lorsqu'on atteint le $p \mathrm{H}$ 4,8 , une floculation nette, et un sérum surnageant jaune verdâtre.

Essai no 16. Précipitation à $40^{\circ}$. - On observe une floculation massive avant que tout l'acide soit ajouté. On en continue néanmoins l'addition, pour être dans les conditions des essais précédents. Le $p H$ du sérum est 4,5.

Tous ces laits sont chauffés $1 / 4$ d'heure à $40^{\circ}$.

Los liqueurs qui étaient homogènes à $0^{\circ}$ et à $10^{\circ}$, donnent des flocons qui adhèrent fortement les uns aux autres. Les masses de caséine précipitées ont un aspect fluide.

Pour les essais 14 et 15 , les produits sont granuleux.

Pour l'essai 16, la masse est très compacte.

Lө chiffre des cendres des caséines ainsi obtenues est donné dans le tableau suivant. Ces dernières ont été lavées par centrifugation deux fois à l'eau distillée, en ayant soin de briser le caillé très finement.

\section{Tableau XIII.}

INFLUENGE DE LA TEMPÉRATURE DE PRÉCIPITATION SUR LE TAUX DE MINÉRALISATION DE LA GASÉINE.

\begin{tabular}{|c|c|c|c|c|}
\hline Essais & $\begin{array}{c}\text { Tempéra- } \\
\text { ture } \\
\text { de } \\
\text { précipita- } \\
\text { tion }\end{array}$ & $\begin{array}{l}\text { Quantité } \\
\text { d'acide } \\
\text { ajouté }\end{array}$ & $\begin{array}{c}\text { pH } \\
\text { du } \\
\text { sérum }\end{array}$ & $\begin{array}{c}\text { Cendres } \\
\text { pour } 100 \mathrm{gr} \text {. } \\
\text { de } \\
\text { caséine } \\
\text { sèche. }\end{array}$ \\
\hline $12 \ldots \ldots \ldots \ldots \ldots \ldots \ldots$ & $0^{\circ}$ & 1,53 & 4,8 & 1,44 \\
\hline $13 \ldots \ldots \ldots \ldots \ldots \ldots \ldots$ & $10^{\circ}$ & " & 4,8 & 1,48 \\
\hline $14 \ldots \ldots \ldots \ldots \ldots \ldots \ldots$ & $20^{\circ}$ & " & 4,8 & 1,70 \\
\hline $15 \ldots \ldots \ldots \ldots \ldots \ldots \ldots$ & $30^{\circ}$ & 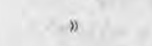 & 4,8 & 1,77 \\
\hline $16 \ldots \ldots \ldots \ldots \ldots \ldots \ldots$ & $40^{\circ}$ & $n$ & 4,5 & 1,95 \\
\hline
\end{tabular}

Comme précédemment, nous trouvons que les caséines sont d'autant plus minéralisées qu'elles sont précipitées à une température plus élevée, toutes conditions étant égales par ailleurs; mais nous n'obtenons pas des différences aussi grandes que celles indiquées précédemment. Cela tient à ce que l'on a opéré sur de petites quantités de lait et que la caséine a été soigneusement disloquée avant le lavage qui a été effectué par centrifugation. Ces conditions ne peuvent pas d'ailleurs être réalisées pratiquement dans l'industrie. 
Dans une autre expérience, un lait est précipité par l'acide chlorhydrique aux températures respectives de : $400,60^{\circ}$ et $\mathbf{8 0}$. - Tandis que précédemment les laits avaient été additionnés de la même quantité d'acide, dans ces essais on ajoute simplement la quantité d'acide nécessaire pour arriver au même $p \mathrm{H}$.

Essai no 17. Température de précipitation $40^{\circ}$. L La quantité d'acide chlorhydrique ajouté, pour avoir un sérum clair, est de $1 \mathrm{gr} .45$ par litre de lait ; le $p$ H du sérum est de 4,82.

Essai no 18. Température de précipitation $60^{\circ}$. - La quantité d'acide chlorhydrique ajouté par litre de lait est de 0 gr. 88 . Le liquide surnageant, blanc verdâtre, a un $p H$ de 4,84 .

Essai no 19. Température de précipitation $80^{\circ}$. - Il faut $0 \mathrm{gr}$. 365 d'acide chlorhydrique par litre de lait pour que la caséine soit précipitée. Le $p H$ du sérum est de 4,82. Las chiffres de cendres sont donnés dans le tableau suivant :

Tableau XIV.

LAIT PRÉCIPITÉ A $40^{\circ}, 60^{\circ}$ ET $80^{\circ}$.

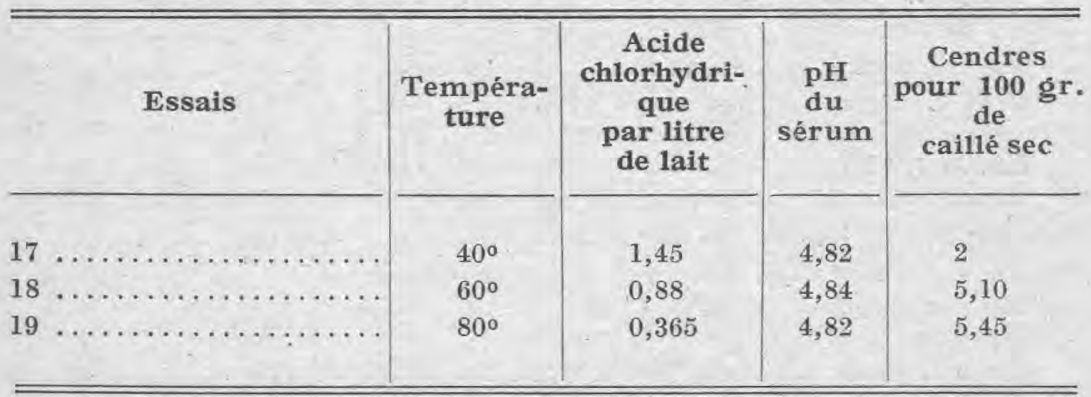

Comme nous le voyons de nouveau dans les deux expériences ci-dessus indiquées, le taux des cendres est dépendant de la température de précipitation; il est d'autant plus grand que cette dernière est plus élevée.

Dans la première expérience pour les températures de $0^{\circ}, 10^{\circ}$, $20^{\circ}, 30^{\circ}$, les laits ont le même $p H$ pour les mêmes quantités d'acide ajouté ; mais, pour la précipitation à $40^{\circ}$, le $p H$ du sérum est nettement inférieur, 4,5 au lieu de 4,8. Ainsi que nous avons déjà eu l'occasion de le dire - mais il est intéressant de le répéter - la précipitation étant obtenue pour une quantité moindre d'acide, et le produit résultant étant plus minéralisé, l'excès d'acide ajouté a simplement joué en influençant le $p H$ du sérum déjà formé.

Dans la seconde expérience, où nous avons opéré les précipitations à $40^{\circ}, 60^{\circ}$ et $80^{\circ}$, les différences se trouvent plus marquées encore. Pour arriver au même $p H=4,8$, il faut des quantités 
d'autant moindres d'acide que la température de précipitation est plus élevée.

En ce qui concerne le taux des cendres, la différence est grande entre les températures de $40^{\circ}$ et de $60^{\circ}$. C'est pourquoi, dans tous les essais industriels qui sont faits à une température dépassant $40^{\circ}$, nous avons remarqué que le chiffre de cendres est toujours très élevé.

\section{Précipitation à des températures différentes d'un caséi- nate de chaux à 600 mmgr. en chaux par litre. - Dans la pré- cipitation des laits par les acides en vue de la fabrication des caséi- nes-acides, nous avons vu l'influence de la température sur la teneur en cendres des caséines obtenues. L'expérience que nous allons rela- ter porte non plus sur le lait, mais sur le caséinate de chaux, elle a pour but de préciser l'influence de la température sur le taux en cen- dres du produit obtenu par acidification. Nous faisons l'essai sur un caséinate à 600 ( 0 gr. 600 de chaux et 30 gr. de caséine par litre), et parallèlement sur le même caséinate chargé en chlorure de calcium.}

Essai n० 20. - Le caséinate à 600 est additionné d'acide chlorhydrique $\mathrm{N} / 10$ a la température ordinaire. Il faut, pour $100 \mathrm{~cm}^{3}$ de caséinate, $15 \mathrm{~cm}^{3}$ d'acide chlorhydrique $\mathrm{N} / 10$, pour obtenir une préeipitation nette, soit 0 gr. 548 par litre. On obtient un sérum opalescent de $p \mathrm{H}=4,8$.

Essai n० 21. - Le même caséinate est chauffé au bain-marie d $60^{\circ}$. Il faut, pour avoir une précipitation de la caséine, $11 \mathrm{~cm}^{3} 5$ seulement d'acide chlorhydrique $\mathrm{N} / 10$ pour $100 \mathrm{~cm}^{3}$ de liqueur, soit 0 gr. 420 par litre. La caséine précipitée est une masse fluide, d'aspect tout différent de celle préparée à la température ordinaire. Le $p$ H du sérum $=5,4$, est blanc laiteux.

Ce qui nous apparaît nettement au cours de ces essais, e'est la difficulté d'obtenir surtout à chaud un sérum très clair. Lorsque nous opérons' avec un lait ou un complexe, le sérum est toujours limpide.

\section{C'est pourquoi nous reprenons la même expérience après addition préalable au caséinate de chlorure de calcium à la dose de 0 gr. 50 au litre.}

Essai no 22 - Pour précipiter à la température ordinaire ce caséinate chargé en $\mathrm{CaCl}^{2}$, il faut $15 \mathrm{~cm}^{2}$ d'acide chlorhydrique $\mathrm{N} / 10$ pour $100 \mathrm{~cm}^{3}$, soit 0 gr. 548 par litre. Dans ce cas, on a un liquide surnageant très limpide et la caséine est précipitée en petits flocons. Le $p$ H du sérum $=4,8$.

Essai no 23. - Le caséinate +0 gr. 5 de $\mathrm{CaCl}^{2}$, est chauffé au bainmarie à $60^{\circ}$. La précipitation devient apparente après l'addition de $2 \mathrm{~cm}^{3}$ seulement d'HCl. On ajoute $12 \mathrm{~cm}^{3} 8$ d'acide chlorhydrique $\mathrm{N} / 10$. On a un produit collant, d'aspect moiré. Le $p H$ du sérum $=4,5$; ce sérum est tout à fait clair. 
Ces caséines précipitées sont lavées par centrifugation à l'eau distillée; après séchage, on détermine le taux des cendres pour chacune d'elles ; il est résumé dans le tableau suivant :

Tablead XV.

PRÉGIPITATION PAR L'ACIDE GHLORHYDRIQUE D'UN GASÉINATE DE GHAUX A DIFFÉRENTES TEMPÉRATURES.

\begin{tabular}{|c|c|c|c|c|c|}
\hline Essais & $\begin{array}{l}\text { Tempé- } \\
\text { rature } \\
\text { de préci- } \\
\text { pitation }\end{array}$ & $\begin{array}{c}\text { HCì en gr. } \\
\text { par litre } \\
\text { de } \\
\text { caséinate }\end{array}$ & $\begin{array}{l}\text { pH du } \\
\text { sérum }\end{array}$ & $\begin{array}{l}\text { Aspect } \\
\text { du sérum }\end{array}$ & $\begin{array}{l}\text { Cen- } \\
\text { dres }\end{array}$ \\
\hline 20 (caséinate 600$) \ldots \ldots \ldots$ & $20^{\circ}$ & 0,548 & 4,8 & opalescent & 1,89 \\
\hline 21 (caséinate 600$). . . \ldots$. & $60^{\circ}$ & 0,420 & 5,4 & blanchâtre & 2,50 \\
\hline $\begin{array}{l}22 \text { (cas. } 600+0 \text { gr. } 5 \text { de } \\
\quad\left(\mathrm{CaCl}^{2}\right) \ldots \ldots \ldots \ldots \ldots\end{array}$ & $20^{\circ}$ & 0,548 & 4,8 & elair & 1,98 \\
\hline 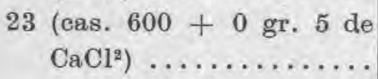 & $60^{\circ}$ & 0,467 & 4,5 & clair & 2,46 \\
\hline
\end{tabular}

Nous voyons que pour l'obtention de la caséine en partant d'un caséinate lorsqu'on opère à $60^{\circ}$, on a une précipitation de la caséine avant que soit atteint le $p H 4,8$; elle se fait au $p H 5,4$, et le produit obtenu est plus minéralisé.

Si nous comparons entre eux les résultats des caséinates chargés en chlorure du calcium, nous voyons qu'en opérant à chaud, le $p H$ du sérum est plus bas que celui de l'opération effectuée à la température ordinaire pour des quantités moindres d'acide ajouté. Malgré ce $p \mathrm{H}$ inférieur, le chiffre de cendres est plus élevé.

Ces résultats sont en tous points comparables à ceux que nous avons obtenus avec le lait. Notons toutefois qu'avec le lait la charge minérale est plus élevée du fait d'un entraînement des sels calciques non complètement dissous.

\footnotetext{
Précipitation d'un caséinate de calcium peu minéralisé, avec des doses différentes de chlorure de calcium et à différentes températures. - Pour expliquer la forte charge minérale des caséines précipitées à chaud, nous avons émis l'hypothèse que dans ces conditions ce n'est pas la caséine qui précipite, mais un caséinate de calcium peu minéralisé. Le $p H$ du sérum importe peu, ou du moins est-il plus exact de dire qu'il ne peut pas nous servir de guide, parce que l'acide que l'on continue d'ajouter n'agit plus sur le caséinate, mais sur le sérum. Pour en instruire la preuve, nous avons été amenée à faire l'expérience qui suit :
} 
Nous préparons un easéinate de faible teneur en chaux ( 0 gr. 400 pour 30 gr. de caséine). Des portions de ce caséinate sont chargées à raison de 1, 2, 3, 4, $5 \mathrm{gr}$. de $\mathrm{CaCl}^{2}$ au litre. Ces liqueurs sont ensuite portées au bain-marie, à différentes températures, et on note leur aspect. Voici ce que l'on observe :

A la température ordinaire. - La liqueur-témoin est homogène ; c'est un liquide opalescent. Les liqueurs chargées en chlorure de calcium paraissent éga lement homogènes, mais, en les transvasant dans un tube z̀ essai, on aperçoit, en faisant couler le liquide sur les bords, des flocons excessivement menus.

A $30^{\circ}$. - Le témoin ne s'est pas modifié sensiblement; pour les caséinates $1,2,3,4,5 \mathrm{gr}$. de $\mathrm{CaCl}^{2}$ au litre, les liqueurs sont laiteuses, maisd'aspect homogène. On aperȩoit cependant de toutes petites masses caillées au fond du tube, si l'on regarde avec attention.

A $40^{\circ}$. - Le témoin n'est pas modifié.

Dans les caséinates $2,3,4 \mathrm{gr}$. de $\mathrm{CaCl}^{2}$ au litre, les caillés sont plus nets, mais les liqueurs surnageantes sont toujours laiteuses.

A $50^{\circ} .-$ Pour les tubes $2,3,4,5 \mathrm{gr}$. de $\mathrm{CaCl}^{2}$ au litre, les caillés sont nettement formés; les liquides surnageants sont opalescents.

Le témoin n'est pas modifié.

Nous répétons la même expérience sur un caséinate encore moins chargé en chaux, à 300 mmgr. par litre. Il est également additionné de $\mathrm{CaCl}^{2}$, à raison de $1,2,3,4,5 \mathrm{gr}$. au litre. Voici ce que nous observons aux différentes températures :

A la température ordinaire $\left(20^{\circ}\right)$. - La liqueur-témoin est opalescente. Les solutions chargées en $\mathrm{CaCl}^{2}$ sont constituées par de tous petits flocons à peine visibles.

A $25^{\circ}$, - La floculation est très nette pour les tubes, $1,2,3,4,5$; le témoin n'est pas modifié.

A $30^{\circ},-$ Le témoin est toujours une liqueur homogène opalescente. Les easéinates à 1, 2, 3, 4, $5 \mathrm{gr}$. $\mathrm{CaCl}^{2}$ sont des liqueurs laiteuses, avec des flocons nets ayant tendance aे se rassembler.

A $35^{\circ}$. - Le témoin a blanchi un peu.

Pour les caséinates à $1,2,3,4,5 \mathrm{gr}$. de $\mathrm{CaCl}^{2}$, les masses caillées sont nettement formées, et se séparent du sérum.

A $40^{\circ}$. - Le témoin n'est toujours pas altéré. Pour les caséinates chargés en $\mathrm{CaCl}^{2}$, les caillés se rassemblent au fond des tubes, les liquides surnageants sont elairs, mais néanmoins opalescents.

A $50^{\circ}$. - Le témoin est une liqueur plus blanche que celle non chauffée, mais non altérée. Les caséinates à $1,2,3,4,5 \mathrm{gr}$. de $\mathrm{CaCl}^{2}$ donnent des caillés très rétractés et des sérums opalescents. 
On arrive à des résultats qui se superposent à ceux de l'expérience antérieure. Nous voyons qu'un caséinate est d'autant plus sensible à l'action combinée de la chaleur et du voisinage des sels calciques solubles qu'il est moins chargé en chaux; c'est du reste ce qu'avait trouvé Ch. Porcher (36). Les conséquences de ces faits vont nous permettre d'expliquer ce qui se passe lors de la précipitation d'un lait par les acides lorsqu'on opère ¿ chaud. L'acide ajouté solubilise la chaux empruntée en partie au phosphate bicalcique et au caséinate pour donner des sels calciques solubles venant s'ajouter à ceux qui préexistent. Quand nous arrivons à un $p H=6$ répondant par conséquent à un easéinate moins calcique que le caséinate originel du lait, nous sommes en présence d'un système chimique ressemblant à ceux que nous venons d'étudier. Nous avons vu que la chaleur facilite la précipitation de caséinates peu calciques en présence d'une charge croissante de sels calciques solubles.

Quand cette précipitation est réalisée, nous pouvons continuer l'addition de l'acide. Ce dernier ne peut agir sur la masse de caséinate précipitée et rétractée, et, comme nous l'avons déjà dit plus haut, il n'intervient que sur le sérum. C'est reconnaître que dans les conditions où l'on effectue la précipitation à chaud, la surveillance de la marche de celle-ci avec le potentiomètre n'a aucune signification.

\section{INFLUENCE DE LA CONCENTRATION DE L'ACIDE EMPLOYÉ.}

Essai no 24. Précipitation à chaud. Agitation lente. Apport rapide d'acide concentré. Caséine non lavée. - La température du lait est de $42^{\circ}$ et l'acide chlorhydrique employé est à $20 \%$, e'est-à-dire plus de 10 fois la concentration de l'acide utilisé dans les essais antérieurs.

5 litres de lait écrémé de $20^{\circ} \mathrm{D}$., $p \mathrm{H}=6,6$, sont portés dans la cuve à double fond à $42^{\circ} \mathrm{C}$. et l'on ajoute l'acide très vite, jusqu'æ̀ ce que la masse de caséine se sépare nettement du sérum que nous obtenons très clair. Le $p H=4,6$. Toute la caséine est ramassée au fond de la cuve, en une masse spongieuse.

Le volume total de l'acide employé est de $35 \mathrm{~cm}^{3}$, soit 1 gr. 40 d'acide par litre de lait.

La caséine est jetée sur un linge et laissée à égoutter toute la nuit. Le lendemain, on la passe à la presse et on la sèche à l'étuve à $40^{\circ} \mathrm{C}$. On obtient des grains compacts teintés en jaune.

(36) Loc. cit., p. 213. 
Tableau XVI.

INFLUENGE DE LA CONGENTRATION DE L'ACIDE EMPLOYÉ SUR LE TAUX DE MINÉRALISATION DE LA GASÉINE.

\begin{tabular}{|c|c|c|c|c|c|c|}
\hline Essais & $\begin{array}{l}\text { Concen- } \\
\text { tration } \\
\text { de l'acide }\end{array}$ & $\begin{array}{l}\text { Tempé- } \\
\text { rature } \\
\text { de préci- } \\
\text { pitation }\end{array}$ & $\begin{array}{c}\text { Acide } \\
\text { chlorhy- } \\
\text { drique } \\
\text { en gr. par } \\
\text { litre de } \\
\text { lait }\end{array}$ & $\begin{array}{c}\mathrm{pH} \\
\text { du } \\
\text { sérum }\end{array}$ & $\begin{array}{l}\text { Cendres } \\
\text { pour } \\
100 \text { gr. de } \\
\text { caséine } \\
\text { anhydre }\end{array}$ & $\begin{array}{c}\text { Rendement } \\
\text { par litre } \\
\text { de lait }\end{array}$ \\
\hline 9 & $2 \%$ & $42^{\circ}$ & 0,96 & 4,60 & 4,36 & 31,40 \\
\hline $24 \ldots \ldots$ & $20 \%$ & $42^{\circ}$ & 1,40 & 4,60 & 4,15 & 31,20 \\
\hline
\end{tabular}

Taux de cendres : $4,15 \%$.

Rendement : 31 gr. 20 au litre.

Nous obtenons des chiffres voisins de cendres, que l'acide soit à $2 \%$ (Essai no 9 ) ou à $20 \%$.

\section{INFL.UENCE DU LAVAGE.}

Essai $n^{\circ}$ 25. Précipitation lente à froid. Caséine bien lavée, jusqu'à non acidité au tournesol. - La température du lait est de $18^{\circ} \mathrm{C}$., la concentration de l'acide : $2,40 \%$; le lait a $18^{\circ} \mathrm{D}$. et son $p \mathrm{H}=6,8$.

On opère sur 5 litres dé lait dans la cuve à double fond, à agitateur mécanique. La précipitation commence ì se faire avec le $p H=5,3$, mais le sérum n'est pas encore clair et la filtration sur papier, d'un essai, est d'une extrême lenteur. On continue l'addition d'acide. Au $p H=4,8$ le filtrat sur papier est clair. On continue néanmoins d'ajouter de l'acide, afin d'arriver au $p H=4,6$.

Le volume total d'acide employé est de $450 \mathrm{~cm}^{3}$, ce qui fait $2 \mathrm{gr} .16$ par litre de lait.

La durée de la précipitation a été d'une heure et demie. Le tout est jeté sur linge et laissé à égoutter jusqu'au lendemain matin. La caséine est bien lavée avec l'eau de la conduite, jusqu'à non acidité au tournesol. Il est à noter que l'égouttage du produit et son lavage sont des opérations lentes.

On a un produit à grains très fins et très blanes. On presse et on laisse à la presse pendant 6 heures environ. Le gâteau est ensuite brisé à la main et séché à l'étuve à $40^{\circ} \mathrm{C}$. On a un produit granulé, à grains fins et blances.

Taux des cendres : $0,90 \%$.

Rendement : 27,30 au litre. 


\section{TABleau XVII.}

INFLUENCE DU LAVAGE SUR LE TAUX DE MINÉRALISATION D'UNE GASÉINE PRÉCIPITÉE A LA TEMPÉRATURE ORDINAIRE.

\begin{tabular}{|c|c|c|c|c|c|c|}
\hline Essais & Lavage & $\begin{array}{c}\text { Tempé- } \\
\text { rature } \\
\text { de } \\
\text { précipi- } \\
\text { tation }\end{array}$ & $\begin{array}{c}\text { Acide } \\
\text { chlorhy- } \\
\text { drique } \\
\text { en gr. par } \\
\text { litre } \\
\text { de lait: }\end{array}$ & $\begin{array}{l}\text { pH } \\
\text { du } \\
\text { sé- } \\
\text { rum }\end{array}$ & $\begin{array}{l}\text { Cendres } \\
\text { pour } \\
100 \mathrm{gr} \text {. } \\
\text { de caséine } \\
\text { anhydre }\end{array}$ & $\begin{array}{l}\text { Rende- } \\
\text { ment } \\
\text { par litre } \\
\text { de lait } \\
\text { écrémé }\end{array}$ \\
\hline & non lavée & $18^{\circ}$ & 2,00 & 4,60 & 2,90 & 31,60 \\
\hline & lavée avec & & & & & \\
\hline & $\begin{array}{c}\text { l'eau de la } \\
\text { conduite }\end{array}$ & $18^{\circ}$ & 2,16 & 4,60 & 0,90 & 27,30 \\
\hline
\end{tabular}

Influence du lavage sur diverses caséines-acides. - Ici, nous avons opéré sur troís caséines préparées en partant du même lait, mais précipitées à la température ordinaire, dans un cas par $\mathrm{HCl}$, dans le second cas par l'acide lactique et, dans le troisième cas, nous avons laissé agir l'acidification naturelle. Les caséines ont êté lavées à l'eau distillée. Voici les résultats obtenus :

Tableat XVIII.

\begin{tabular}{|c|c|c|c|}
\hline & \multicolumn{3}{|c|}{$\begin{array}{c}\text { Taux des cendres } \% \text { sur le produit } \\
\text { anhydre }\end{array}$} \\
\hline & HCl & $\begin{array}{c}\text { s précipit } \\
\text { Acide } \\
\text { lactique } \\
\text { (apporté) }\end{array}$ & $\begin{array}{l}\text { s par : } \\
\text { Acide lacti- } \\
\text { que (Acidi- } \\
\text { fication } \\
\text { naturelle) }\end{array}$ \\
\hline Non lavée .... & 2,66 & 1,24 & 3,04 \\
\hline Lavée $\ldots \ldots \ldots \ldots \ldots \ldots \ldots \ldots$ & 0,97 & 0,45 & 0,92 \\
\hline
\end{tabular}

Influence du lavage et de la température de précipitation sur la minéralisation de la caséine-acide. - L'acide employé est de l'acide chlorhydrique $\mathrm{N} / 2$.

On effectue une précipitation du lait à la température ordinaire et une autre à $50^{\circ}$.

Le caillé de l'une et l'autre de ces opérations est recueilli et égoutté. On en fait deux parts : l'une n'est pas lavée et l'autre est lavée avec de l'eau distillée. 
Voici quels sont les résultats obtenus en ce qui concerne les cendres, dans ces quatre circonstances différentes :

Tableat XIX.

INFLUENGE DU LAVAGE ET DE LA TEMPÉRATURE DE PRÉCIPITATION SUR LA MINÉRALISATION DE LA GASÉINE ACIDE.

\begin{tabular}{|c|c|c|}
\hline \multirow[b]{2}{*}{ 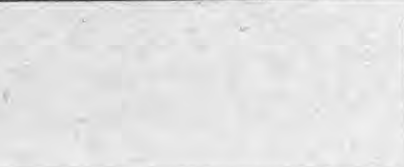 } & \multicolumn{2}{|c|}{ Cendres $\%$ sur le produit anhydre } \\
\hline & $\begin{array}{l}\text { Précipitation à } \\
\text { la température } \\
\text { ordinaire }\end{array}$ & Précipitation à $50^{\circ}$ \\
\hline Caséine non lavée ..... & 3,68 & 4,94 \\
\hline Caséine lavée ............ & 1,24 & 3,96 \\
\hline
\end{tabular}

Deux points sont à relever dans cette expérience : d'abord le taux plus élevé des cendres quand on opère à chaud, ensuite l'influence du lavage ; mais encore celavage joue-t-il moins sur la déminéralisation lorsque la précipitation a lieu à $50^{\circ}$ que lorsqu'elle se fait à la température ordinaire. A $50^{\circ}$ le lavage détermine une déminéralisation de $1 \%$ alors qu'elle atteint 2,50\% pour la caséine précipitée à la température ordinaire. Cela tient à ce que nous précipitons un caséinate (peu minéralisé il est vrai) mais non une caséine libre de toute charge, et que d'autre part nous avons affaire à un produit se présentant en une masse compacte qui retient du sérum dans ses mailles et qui ne se laisse pas pénétrer par l'eau.

\section{CONCLUSIONS.}

Influence de la rapidité de l'apport de l'acide. - La rapidité plus ou moins grande de l'apport de l'acide ne paraît pas avoir une très grande importance. Elle doit être cependant combinée avec l'agitation de façon à n'avoir pas une relative concentration de l'acide en un point de la cure.

L'influence de l'agitation. - L'agitation, qu'elle soit lente ou rapide, n'a pas beaucoup d'influence sur le taux des cendres et le rendement. Nous n'avons travaillé que sur de petites quantités de lait, si bien que ce que nous appelons une agitation lente est, néanmoins, assez bonne, comparée à ce qu'on obtiendrait dans l'industrie avec des cuves de 500 ou 1.000 litres, et même davantage.

Nous conseillons d'agiter, au début, assez énergiquement, pour répartir très régulièrement l'acide apporté dans la masse et de ralentir ensuite, pour saisir plus facilement la fin de la préeipitation et obtenir un grain de la grosseur désirée, lequel, si l'agitation était 
trop vive, serait trop menu. Le produit obtenu filtrerait mal et il y aurait des pertes dans le rendement.

L'influence de la température. - La température à laquelle on opère la précipitation est un facteur important, celui qui agit le plus sur le taux des cendres. Celui-ei est, en effet, très différent, selon que la précipitation se fait à la température ordinaire ou à chaud. Nous avons dans ce cas un caséinate de chaux et non de la caséine pure complètement déminéralisée. La précipitation à chaud est donc d'une mauvaise technique.

Influence du lavage. - Une caséine précipitée à chaud, même lavée, a un chiffre de cendres très élevé, tandis qu'une caséine précipitée à froid, et lavée, a un taux minéral nettement plus bas. Celle-là se lave mal, celle-ci se lave bien.

Le lavage enlève les acides qui se sont fixés sur la caséine, mais il déminéralise peu le produit fabriqué si la caséine a été précipitée à chaud $\left(40^{\circ}\right.$ et $\left.50^{\circ}\right)$ comme cela est pratiqué si souvent dans l'industrie.

Nous devons insister sur ce que la caséine, précipitée à froid, filtre difficilement sur toile et se presse mal. Les opérations sont plus longues. Il y a des pertes dans le rendement. C'est pourquoi nous allons essayer de cuire le grain, tant pour le raffermir et le faire se rétracter sur lui-même - ce qui contribue à exsuder une partie du sérum qu'il renferme -, que pour faciliter la filtration du sérum et le pressage du caillé.

Ici, les précipitations sont faites, comme dans les essais précédents, avec de l'acide chlorhydrique.

\section{CASÉINE CHAUFFÉE DANS SON SÉRUM.}

\section{Essai no 26. Caséine précipitée à froid. Cuisson dans le sérum.} Pas de lavage. - Le lait a une acidité de $22^{\circ} \mathrm{D}$. L'a cide employé est à $3 \%$. On opère sur 5 litres de lait. On ajoute l'acide lentement, l'agitation étant environ de 80 tours à la minute. Il a fallu $345 \mathrm{~cm}^{3}$ d'acide pour arriver au $p H 4,7$, soit 2 gr. 07 d'acide par litre de lait.

On continue l'agitation, tout en la ralentissant pendant le chauffage ; on porte gradusllsm nnt la masse $\grave{a} 50^{\circ}$ et on maintient 10 minutes à cette température. On a un proluit en grains très fins, légèrement teinté. On décante le sérum, on égoutte la caséine sur un linge et on la passe à la presse. Déjà, sur linge, le produit donne la sensation d'être plutôt see; aussi se brise-t-il faeilement et, à la sortie de la presse, il se résoud, à la main, en poudre fine. On sèche à $40^{\circ}$ et on obtient un proluit granulé qui se brise facilement ; il est légèrement teinté en jaune.

Taux des cendres : $1,60 \%$.

Rendement : 29,2 .

Acide : 1,16\%. 
Essai no 27. Même essai qu'en 8. - On opère dans les mêmes conditions que dans l'essai précédent, avec 5 litres de lait. Il faut $340 \mathrm{~cm}^{3}$ d'acide pour arriver au $p \mathrm{H}=4,7$, soit $2 \mathrm{gr}$. 04 d'acide par litre de lait.

La caséine est euite dans son sérum, mais, cette fois, à $60^{\circ}$, et enmaintenant 15 minutes cette température. La moitié est prélevée. On obtient une caséine en poudre, légèrement teintée, que l'on passe à la presse à main. Séchée à l'étuve à $40^{\circ}$, elle a le mĉme aspect que la caséine précédente.

Taux des cendres : $2,06 \%$.

Acidité : 1,17 .

Notons que le taux des cendres est plus élevé que dans le cas précédent. Cela provient peut-être de ce que l'on a cuit à $60^{\circ} \mathrm{au}$ lieu de $50^{\circ}$. D'autre part, les caséines n'ayant pas été lavées, cela peut provenir également des conditions du pressage.

TableaU XX.

INFLUENGE DE LA GUISSON SUR LE TAUX DE MINÉRALISATION DE LA CASÉINE.

\begin{tabular}{|c|c|c|c|c|c|c|}
\hline Essajs & $\begin{array}{c}\text { Cuisson } \\
\text { dans le sérum } \\
\text { (température } \\
\text { et } \\
\text { durée) }\end{array}$ & $\begin{array}{l}\text { Tempé- } \\
\text { rature } \\
\text { de } \\
\text { précipi- } \\
\text { tation }\end{array}$ & $\begin{array}{l}\text { Acide } \\
\text { chlor- } \\
\text { hydrique } \\
\text { en gr. } \\
\text { par litre } \\
\text { de lait }\end{array}$ & $\begin{array}{l}\text { pH } \\
\text { du } \\
\text { sé- } \\
\text { rum }\end{array}$ & $\begin{array}{l}\text { Cendres } \\
\text { pour } \\
100 \text { gr. } \\
\text { de } \\
\text { caséine } \\
\text { anhydre }\end{array}$ & $\begin{array}{l}\text { Rendement } \\
\text { par } \\
\text { litre de lait }\end{array}$ \\
\hline $26 \ldots$ & 10 minutes à $50^{\circ}$ & $18^{\circ}$ & 2,07 & 4,70 & 1,60 & 29,20 \\
\hline $27 \ldots$ & 15 minutes à $60^{\circ}$ & $18^{\circ}$ & 2,04 & 4,70 & 2,06 & \\
\hline $7 \ldots$ & non cuite & $18^{\circ}$ & 2,00 & 4,60 & 2,90 & 31,60 \\
\hline $8 \ldots$ & non cuite & $18^{\circ}$ & 1,89 & 4,80 & 2,84 & 31,60 \\
\hline
\end{tabular}

\section{Influence du lavage.}

Essai no 28. Les conditions sont les mêmes que dans l'opération précédente, mais la seconde moitié de la caséine, préparée comme il a été dit dans l'essai antérieur, a été lavée deux fois à l'eau distillée, dans la cuve même, après cuisson, en opérant par décantation. Elle est ensuite égouttée sur linge et pressée. La caséine se trouve sous la forme d'une poudre. Le gâteau de pressage se défait très bien à la main.

Taux des cendres : $0.34 \%$.

Acidité : 0,48 .

Il est intéressant de comparer ces chiffres à ceux de l'essai antérieur qui ne comportait pas de lavage. Nous constatons une diminution considérable du taux des cendres ainsi que de celui de l'acidité, et la belle couleur du produit, après séchage.

Essai n० 29. Gaséine précipitée à froid. Ghauffage au sein du sérum. Deux lavages avec 1'eau de la conduite. - L'acide employé a une concentration de $2,4 \%$. Le lait écrémé a $25^{\circ} \mathrm{D}$. et 
un $p \mathrm{H}$ de 6,50. La température à laquelle la précipitation a lieu est de $18^{\circ} \mathrm{C}$.

5 litres de lait sont agités dans la cuve à double fond. L'acide est ajouté très lentement. $\mathrm{Au} p \mathrm{H}=5$, on ne note pas encore de précipitation. $\mathrm{Il}$ faut arriver au $p \mathrm{H}=4,9$ pcur observer l'apparition des premiers flocons. On laisse pendant 3 həures aे ce $p$ H, puis, au bout de ce temps, on continue l'addition de l'acide pour arriver au $p$ H $=4,55$.

Volums de l'acide employé : $430 \mathrm{~cm}^{3}$, soit 2 gr. 06 d'acide par litre de lait.

$L_{a}$ caséine est ensuitè cuite dans le sérum à $45^{\circ}$. On a mis une heure et demie pour atţindre cette tımṕ́ratura. La caséine se rassemble. On décante le sérum et on ajoute de l'eau de la conduite, 5 litres environ. On agite à nouveau et on lave ensuite, deux fois, par décantation. La caséine est égouttée sur toile. Elle se présente en grains fins et très blanes. Elle se presse facilement et le gâteau se brise aisémэnt à la main. Séchée à l'ótuve à $40^{\circ}$, on a un produit granulé, blane, à grains fins.

Taux des cendres: $1,31 \%$.

Rendement: 30 gr. au litre.

En comparant le chiffre de cendres avec celui de l'essai antérieur, on voit l'intérêt qu'il y a à effectuer le lavage avec de l'eau distillée qui, dans l'industrie, peut être fournie par les eaux de condensation.

Essai no 30. Même essai que le précédent. Précipitation à froid. Gaséine chauffée dans son sérum et lavée deux fois avec l'eau de la conduite.

Cendres: $1.40 \%$.

Acidité: 0,29 .

Rendement: 28 grammes au litre.

Essai no 31. Mêmes opérations que dans 1'essai précédent. On s'arrête à 1'obtention d'un sérum clair dans la précipitation, sans chercher à atteindre le point isoélectrique. - Le lait, très acide, a $28^{\circ} \mathrm{D}$.; le $p \mathrm{H}=6,40$.

On opère sur 5 litres de lait. Il faut $150 \mathrm{~cm}^{3}$ d'acide chlorhydrique à $3 \%$ pour arriver à un sérum clair, soit $0 \mathrm{gr} .900$ d'acide par litre, et le $p$ H, à ce moment, est de 5,00 .

La casóine est cuite dans son sérum à $50^{\circ}$; on maintient 10 minutes à cette tempśrature, on décante, on lave deux fois par décantation avec l'eau de la conduite. Commэ précédemmont, on obtient un produit en grains fins et blanes. La caséine est laissée à égoutter toute la nuit; elle est passée à la presse le lendemain et séchée à $40^{\circ}$. Le produit est granulé, blanc.

Cendres: $2 \%$

Acidité: 0,38 .

Rendement: 27 grammes au litre.

Le taux des cendres a légèrement augmenté par rapport à ce qu'il était dans les deux essais qui précèdent. Cela est dû à la moindre quantité d'acide employé pour la précipitation. 


\section{TABLEAU XXI.}

GASÉINE CUITE DANS SON SÉRUM. INFLUENCE DU LAVAGE SUR LE TAUX DE MINÉRALISATION DE LA GASÉINE.

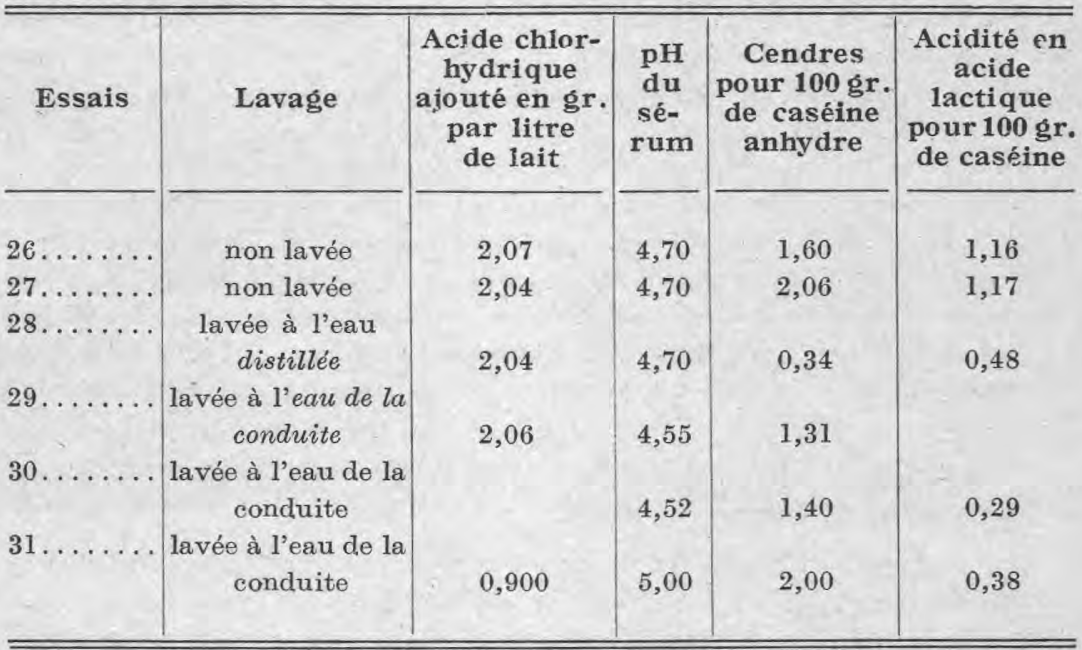

Caséine précipitée à la température ordinaire, cuite dans son sérum à $50^{\circ}$. - L'acide précipitant est $\mathrm{HCl} \mathrm{N/2}$. Ici, la précipitation s'est faite à la température ordinaire et on a ensuite porté graduellement à la température de $50^{\circ}$. Voici les résultats obtenus :

Tableau XXII.

\begin{tabular}{|c|c|c|}
\hline & \multicolumn{2}{|c|}{$\begin{array}{c}\text { Cendres } \% \text { sur le produit } \\
\text { anhydre }\end{array}$} \\
\hline & Non cuite & $\begin{array}{l}\text { Cuite dans son } \\
\text { sérum }\end{array}$ \\
\hline Caséine non lavée & 2,61 & 0,89 \\
\hline Caséine lavée à l'eau distillée & 0,21 & 0,26 \\
\hline
\end{tabular}

La grande différence qu'il y a dans le taux de la minéralisation entre la caséine non lavée, cuite et non cuite dans son sérum ne signifie pas que la cuisson joue un rôle plus ou moins déminéralisateur. La cuisson a pour but, comme nous le savons, - selon l'expression de la fromagerie - de ressuyer le grain, de le faire se contracter en expulsant le sérum qu'il renferme.

Nous voyons, d'autre part, le rôle très important du lavage sur les deux sortes de caséine : la non cuite et la cuite. Il est considé- 
rable dans un cas comme dans l'autre, mieux marqué sur la première, puisque la caséine non euite et non lavée est plus riche en matières minérales.

Bien que nous ne notions pas de différence dans le taux des cendres entre la caséine lavée cuite et non cuite, la cuisson est néanmoins à recommander, elle facilite énormément le travail du grain (filtration et lavage).

Caséine cuite dans la deuxième eau de lavage. - Essai. no 32. Précipitation à froid. Caséine chauffée dans la deuxième eau de lavage (Il s'agit de l'eau de la conduite). - Le lait a $18^{\circ}$ D. ; $p \mathrm{H}=6,70$. L'acide employé est à $2,4 \%$. La température de précipitation est de $18^{\circ} \mathrm{C}$.

On opère sur 5 litres de lait. Pour arriver au $p H 4,6$, il faut $400 \mathrm{~cm}^{3}$ d'acide, soit 1 gr. 92 d'acide par litre. L'addition de l'acide a duré une heure. Pendant 2 houres, on laisse déposer la caséine, puis on décante le sérum clair. On ajoute de l'eau de la conduite : 5 litres environ. On décante après avoir agité la caséine et laissé cette dernière se rassembler à nouveau. On ajoute, une deuxième fois, de l'eau de la conduits et l'on chauffe graduellement à $50^{\circ}$, ce qui demande une $1 / 2$ hэure. Quand cette tempórature est atteinte, on agite pendant 10 minutes. On laisse ensuite repozer et l'on décante le liquide surnageant qui est de couleur jaunâtro. La caséine forme un bloc très ramassé. On la presse et on la brise ensuite à la main. Lə grain est très serré et le gâteau se défait difficilement. La caséine est ensuite séchée à $40^{\circ}$.

Taux des cendres: $1,10 \%$.

Acidité : 0,19 .

Rendement : 27 gr. 90 au litre.

Essai no 33. Mêmes conditions opératoires que dans 1'essai précédent, mais on emploie de l'acide plus concentré $(\mathrm{HCl}$ à $20 \%$ ). - Le lait a $22^{\circ}$ D. L'acide est ajouté lentement. On opère sur 5 litres. Il faut $46 \mathrm{~cm}^{3}$ d'acide pour arriver au $p \mathrm{H}=4,8$, soit 1 gr. 84 d'acide par litre de lait.

La caséine obtenue est en grains très fins, mais qui se rassemblent néanmoins. On décante le sérum et on lave deux fois, comme précédemment. Puis la caséine est chauffée à $57^{\circ}$ dans la deuxième eau de lavage. Cette température est maintenue pendant 10 minutes. La caséine forme une masse élastique et spongieuse. On la passe à la presse et on l'y laisse toute la nuit. Elle est ensuite brisée à la main et séchée à $40^{\circ}$.

Cendres: $1,46 \%$.

Acidité: 0,28 .

Rendement: 30 gr. 20 . 
Nous voyons que la caséine cuite dans l'eau de lavage a un aspect tout différent de celle qui a été cuite dans le sérum. Alors que cette dernière donne, après pressage, un gâteau dont les grains se séparent bien les uns des autres, la caséine cuite dans l'eau de lavage forme une masse compacte, collante, difficile à désagréger et, par conséquent, de séchage difficile. Aussi, allons-nous faire un essai en cuisant la caséine, non plus dans l'eau de la conduite, qui est voisine de la neutralité, mais dans une eau acide de $p \mathrm{H}=4,7$.

Essai $n^{\circ}$ 34. Précipitation à froid. Caséine chauffée dans la deuxième eau de lavage, qui est une eau distillée chlorhydrique, de $p H$ voisin de 4,7 . - Lait $22^{\circ} \mathrm{D}$. L'acide employé est à $3 \%$. On opère sur 5 litres de lait, dans une capsule de porcelaine et non plus, cette fois, dans la cuve émaillée à double fond. On agite à la main, en réalisant toutefois une bonne agitation. L'acide est ajouté lentement. On obtient un sérum clair pour un $p \mathrm{H}=5,00$. On laisse pendant 2 heures. On continue ensuite l'addition d'acide pour arriver au $p \mathrm{H}=4,7$.

Volume d'acide nécessaire : $350 \mathrm{~cm}^{3}$, soit $2 \mathrm{gr}$. 10 par litre de lait.

Le sérum est très limpide et la caséine se dépose au fond de la capsule. On laisse reposer 2 heures et la décantation du sérum est facile. On lave à froid avec l'eau de la conduite, 5 litres environ. On décante. On ajoute le même volume d'eau distillée chlorhydrique au $1 / 100.000$, ce qui réalise un $p H$ voisin de 4,7. On chauffe à $50^{\circ}$ pendant 10 minutes. La caséine reste en grains qui ne s'agglomèrent pas. Comme nous l'avons noté dans l'essai précédent, la caséine est égouttée sur linge, ce qui se fait très rapidement. Elle est ensuite passée à la presse. Le gâteau se défait très bien à la main en petits grumeaux.

Cendres: $0,28 \%$.

Acidité: 0,19 .

Rendement: 27 gr. 4.

Essai no 35. Même opération que la précédente. - La caséine est chauffée dans la première eau de lavage qui est une eau distillée chlorhydrique de $p \mathrm{H}$ voisin de 4,7.

On opère sur 5 litres de lait. L'acide employé est à $3 \%$. Il faut $340 \mathrm{~cm}^{3} \mathrm{~d}$ 'acide pour arriver au $p H=4,7$, soit 2 gr. 04 d'acide par litre de lait. Au bout de 2 heures, le sérum est décanté ; on ajoute 5 litres d'eau distillée chlorhydrique dont la con. centration est de $1 / 100.000$. On cuit à $50^{\circ}$ et on laisse à cette température pendant 10 minutes. On décante. Le sérum se sépare bien; la caséine est en petits grains qui ne s'agglomèrent pas. Elle est ensuite laissée toute la nuit sous la presse. On sèche à $40^{\circ}$ et on obtient un produit granulé, un peu plus teinté que le précédent.

Cendres: $0,84 \%$.

Rendement: 27 gr. 4 .

Dans tous les essais de cette série, on a agité pendant la cuisson. 
TableaU XXIII.

GASÉINE GUITE DANS LA DEUXIÈME EAU DE LAVAGE.

INFLUENGE DE LA NATURE DE L'EAU EMPLOYEE POUR LA GUISSON SUR LA MINÉRALISATION DE LA GASÉINE.

\begin{tabular}{|c|c|c|c|c|c|c|}
\hline Essais & $\begin{array}{l}\text { Eau } \\
\text { employée } \\
\text { pour } \\
\text { la cuisson }\end{array}$ & $\begin{array}{l}\text { Acide } \\
\text { chlorhy- } \\
\text { drique } \\
\text { en gr. } \\
\text { par litre } \\
\text { de lait }\end{array}$ & $\begin{array}{l}\text { pH } \\
\text { du } \\
\text { sé- } \\
\text { rum }\end{array}$ & $\begin{array}{l}\text { Cendres } \\
\text { pour } \\
100 \mathrm{gr} \text {. } \\
\text { de caséine } \\
\text { anhydre }\end{array}$ & $\begin{array}{c}\text { Acidité } \\
\text { en acide } \\
\text { lactique } \\
\text { pour } \\
100 \text { gr. de } \\
\text { caséine }\end{array}$ & $\begin{array}{l}\text { Rende- } \\
\text { ment par } \\
\text { litre } \\
\text { de lait }\end{array}$ \\
\hline $\begin{array}{ll}32 & \ldots \\
33 & \ldots\end{array}$ & $\begin{array}{l}\text { eau de la conduite } \\
\left(2^{\mathrm{e}} \text { eau de lavage }\right) \\
\text { eau de la conduite }\end{array}$ & 1,92 & 4,60 & 1,10 & 0,19 & 27,90 \\
\hline $34 \ldots$ & $\begin{array}{c}\left(2^{\mathrm{e}} \text { eau de lavage }\right) \\
\text { eau distillée } \\
\text { chlorhydrique } \\
\text { au } 1 / 100.000 \\
\left(2^{\circ} \text { eau de lavage }\right) \\
\text { eau distillée } \\
\text { chlorhydrique } \\
\text { au } 1 / 100.000 \\
\left(1^{\text {re } e a u ~ d e ~ l a v a g e ~}\right)\end{array}$ & 2,10 & 4,80 & 1,46 & 0,28 & 30,20 \\
\hline
\end{tabular}

conclusıons. - Caséine cuite dans son sérum. - Le procédé qui consiste à précipiter la caséine à froid et à la cuire dans son sérum donne de très bons résultats. Le chiffre des cendres est peu élevé; le rendement est bon. Le lavage avec l'eau de la conduite diminue peu le chiffre des cendres, mais notablement celui de l'acidité. Si le lavage se fait avec de l'eau distillée, le taux de minéralisation diminue considérablement; l'acidité diminue également, mais il n'y a pour ainsi dire pas de différence sensible, de ce côté, avec l'acidité résultant du lavage avec l'eau de la conduite, ce qui se conçoit fort bien. L'eau de la conduite apporte de la chaux et, dans ces conditions, la diminution du taux minéral de la caséine du produit fabriqué peut être légère, alors que, si le lavage se fait avec de l'eau distillée, il n'en est plus de même. Quant à l'acidité, elle disparaît aussi bien avec l'eau distillée qu'avec l'eau de la conduite.

Si nous comparons l'essai $n^{\circ} 31$ aux essais $n^{\circ} 29$ et $n^{\circ} 30$ dans lesquels la précipitation avait été faite à la température ordinaire, à des $p H$ différents, nous voyons que la caséine des deux derniers a un taux minéral inférieur à celui de la caséine du premier : 1,31 et 1,40 , avec des $p H$ respectivement de 4,55 et 4,52 , alors que l'on a $2 \%$ de cendres avec un $p \mathrm{H}$ de 5,00 (essai $\mathrm{n}^{\circ} 31$ ). 
Caséine cuite dans l'eau de lavage. - On observe des différences dans l'aspect de la caséine obtenue; le produit est collant et de dessiccation difficile.

Caséine cuite dans une eau de lavage de $p \mathrm{H}=\mathbf{4 , 7}$. Nous obtenons un chiffre de cendres tout à fait intéressant et l'acidité est faible. Le produit ne colle pas.

\section{COMPARAISONS ENTRE DES CASÉINES PRÉCIPITÉES DANS LES MÊMES CONDITIONS, A DES pH DIFFÉRENTS.}

Nous avons eu, dans des essais antérieurs, l'occasion de noter l'influence du $p \mathrm{H}$ sur la minéralisation des easéines. Nous allons faire trois essais en opérant avec des $p$ H respectivement : 5,0, 4,7, 4,0.

La précipitation se fait à froid avec une bonne agitation et un apport lent d'acide; la caséine est cuite dans son sérum et lavée.

L'acide employé est de l'acide chlorhydrique à $2,4 \%$; le lait, très acide, de $p \mathrm{H}=6,25$, ̀̀ $35^{\circ} \mathrm{D}$.

Essai n ${ }^{\circ}$ 36. Le $p \mathrm{H}$ auquel on s'arrête est 5,00. - Le sérum est laiteux, la précipitation est commençante. On n'aperçoit que quelques flocons seulement. Le volume d'acide employé pour 5 litres est de $240 \mathrm{~cm}^{3}$, soit $1 \mathrm{gr}$. 15 par litre de lait. On chauffe à $50-55^{\circ} \mathrm{et}$ on maintient cette température pendant 10 minutes. La caséine se sépare bien du sérum et elle se rassemble en une masse compacte. Le sérum est elair et son $p \mathrm{H}$ est de 5,00 . Le sérum est décanté et la caséine lavée à froid, deux fois, avec l'eau de la conduite. Elle est passée à la presse et séchée à $40^{\circ}$. Elle est teintée en jaune. On a un produit non homogène, constitué par un mélange de grains compacts luisants, d'autres ayant un aspect plutôt cristallin.

Taux des cendres: $2,22 \%$.

Acidité: 0,58 .

Rendement: 31 gr. $\%$.

Essai no 37. Le $p H$ du sérum est 4,7, c'est-à-dire le point isoélectrique de la caséine. Le sérum est extrêmement clair et la précipitation est d'une grande netteté. Il faut $350 \mathrm{~cm}^{3}$ d'acide, soit $1 \mathrm{gr}$. 68 d'acide par litre de lait. La caséine est cuite dans son sérum à $50^{\circ}-55^{\circ}$ et on maintient cette température pendant 10 minutes, comme dans l'essai précédent. Le $p H$ du sérum, après la cuisson, est de 4,7. Séchée à $50^{\circ}$, on a un produit très blanc et finement granulé.

Taux des cendres : $1,12 \%$.

Acidité : 0,20 .

Rendement : 29 gr. 8 par litre.

Essai no 38. Le $p \mathrm{H}$ atteint lors de la précipitation est de 4.La caséine se rassemble bien au fond de la capsule. Il faut $415 \mathrm{~cm}^{3}$ d'acide pour arriver au $p H=4$, soit 2 gr. d'acide par litre. Le grain est cuit comme dans les 
deux essais antérieurs et toutes les opérations ultérieures sont les mêmes. La caséine, séchée à $50^{\circ}$, donne un produit granulé, blanc, qui se pulvérise à la main.

Cendres : $0,24 \%$.

Acidité : 0,20.

Rendement : 30 gr. 3 .

Tablead XXIV.

GASÉINES PRÉCIPITÉES DANS LES MEMES GONDITIONS A DES $\not H$ DIFFÉRENTS.

\begin{tabular}{|c|c|c|c|c|c|}
\hline Essais & $\begin{array}{l}\text { pH du } \\
\text { sérum }\end{array}$ & $\begin{array}{l}\text { Acide chio- } \\
\text { rhydrique } \\
\text { en gr. } \\
\text { par litre } \\
\text { de lait }\end{array}$ & $\begin{array}{l}\text { Cendres } \\
\text { pour } 100 \text { gr } \\
\text { de caséine } \\
\text { anhydre }\end{array}$ & $\begin{array}{c}\text { Acidité } \\
\text { en acide } \\
\text { lactique } \\
\text { pour } 100 \text { gr. } \\
\text { de caséine }\end{array}$ & $\begin{array}{l}\text { Rendement } \\
\text { par litre de } \\
\text { lait écrémé }\end{array}$ \\
\hline 36 . & 5 & 1,15 & 2,22 & 0,58 & 31,3 \\
\hline $37 \ldots \ldots \ldots$ & 4,7 & 1,68 & 1,12 & 0,28 & 29,8 \\
\hline $38 \ldots \ldots \ldots \ldots$ & 4,0 & 2,00 & 0,24 & 0,20 & 30,3 \\
\hline
\end{tabular}

conclusions. - Le chiffre des cendres est plus élevé pour la caséine qui a été précipitée à un $p H$ de 5 , donc au-dessus du point isoélectrique. Ces résultats s'expliquent fort bien si on compare les quantités d'acide ajoutées par litre de lait. Pour la caséine précipitée au $p H 4$, on obtient un produit dont le taux de cendres est extrêmement bas ; le rendement est excellent et l'acidité peu élevée. Nous notons l'aspect de ce produit qui se pulvérise facilement à la main et l'on se demande s'il ne serait pas préférable, lors de la précipitation de la caséine, de descendre au-dessous du point isoélectrique.

(A suivre).

\title{
CONTRIBUTION \\ A L'ÉTUDE DE LA LACTATION HUMAINE
}

\author{
par \\ Mme C. VINCENT et \\ Chef des travaux \\ M. J. VIAL \\ Assistant
}

du Laboratoire de Physiologie de la Faculté de Médecine de Lyon.

(Suite.)

g) INFLUENCES DIVERSES POUVANT RETENTIR SUR LA QUANTITE

DE LAIT SÉCRÉtEe. - Au cours de nos recherches, nous avons eu l'occasion d'observer l'influence d'un certain nombre de facteurs sur la sécrétion lactée.

Pour que ces observations aient une valeur, il est nécessaire, bien entendu, que chaque facteur (psychique, alimentaire, etc.) 